\title{
Research on the Network Security Management of Artwork E-commerce in New Media Context
}

\author{
Yanli Li \\ Yunnan Arts University, No.1577 Yuhua Road,Chenggong District,Kunming 650500,Yunnan \\ Province,P.R.China \\ lyledu@qq.com
}

Keywords: New Media; artwork e-commerce; Network Security; Security Control

\begin{abstract}
Starting from the concept of new media and its era background, this paper expounded the connotation of artwork e-commerce, analyzed the security issues faced by artwork e-commerce, its network security control and network security strategies were introduced in detail, and an effective management mode about network security of artwork e-commerce came into being, which leads and promotes the rapid and healthy development of artwork e-commerce in china.
\end{abstract}

\section{Introduction}

With the popularity of new media technology, the segmentation of e-commerce and the development of art trading, the traditional art market has changed fundamentally. E-commerce plays a more and more important role in the field of art trading. So artwork e-commerce emerges as the times require. It has changed the operation mode of the traditional art business, and has gradually become a remarkable new mechanism of art trading because of the higher efficiency of art business and the lower cost of art trading. The artwork e-commerce has become an inevitable choice for Arts and culture industries in China integrate into the global economy, as well as causing widespread concern of researchers and consumers. However, the open and interactive features of the Internet has brought many security issues, which has become a bottleneck for the further development of artwork e-commerce. How to perfect the network security management, set up a safe and reliable application environment, has become a key issue affecting the development of artwork e-commerce.

\section{Various opinions: The coming of new media era}

The new media is called compared to the traditional media, whose concept was firstly proposed in 1967 by P. Goldmark, director of the technology research institute in Columbia broadcasting System (CBS) of USA. It refers to a new media form developed after those traditional media, such as newspapers, broadcast and television. By using the digital technology, network technique and mobile technology, the new media, forms of communication and media, can provide information and entertainment services to users through the channels of Internet, wireless communication network and satellite as well as the terminals of computer, mobile phone and digital TV. Strictly speaking, the new media should be called digital media. Xiong Chengyu, a professor of Tsinghua University, said that: "the new media is a constantly changing concept, which extends on the base of today's network. The problems of wireless mobile, the appearance of other new media forms and something related to computer, all these can be called new media.

The fifth information revolution, breaking out in the 20th century, was marked by the application of computers and resulted in the birth of digital media represented by Internet, so it is also called "new media revolution" or "digital revolution". Its rapid development had led to the development and application of the network technology and communication technology, affecting all areas of social life, art is one of them. As a new channel for information communication and Product Sales, the new media created a new business environment for enterprises, the relationship between enterprises and customers, partners and competitors will be reconstructed. 


\section{Clear positioning: artwork e-commerce under the concept of refined e-commerce}

Today is the heyday of e-commerce, in the case of JD.COM and VANCL are members of B2C and Taobao is part of $\mathrm{C} 2 \mathrm{C}$, there are still many dark corners neglected by e-commerce giants. Under the condition of unprecedented competitors and capital gradually concentrate to the superior enterprises, the refined e-commerce begin to enter the public views. It contains two meanings: the first is the segmentation of commodities and the difference between marketing strategies, for instance, JD.COM sells all 3C digital products, and you only sell digital SLR cameras, with the smaller coverage of people and more centralized resources, so you can more easily attract the target users. The second is to refine the additional service of commodities, because of large online stores should meet the needs of various goods and different users, so if you can provide some detailed functions that users are eager for, the result is naturally efficient with half of effort. Such as goods receipt messages, micro-blog and IM customer service. Based on this, the artwork e-commerce, tourism e-commerce, garment e-commerce are all living examples of the refined e-commerce.

Artwork e-commerce, this unique business model, its connotation has a strong meaning of deconstruction if we look from the deep. About this point, as long as we put it together with the contexts of "new media", "globalization" and "network economy", it is easy to see. So, we should understand and review it with a new perspective, perhaps we can put it this way: The current art business, being a fission state caused by Internet and other new media, is a transformation which can be traced back to the original. The changes about the context and state of art business are also paradigm's shifts of that. Artwork e-commerce in the new media era will undoubtedly become the new paradigm of art business.

\section{Targeted: security control in E-Business of Artwork}

The business security is the core and key of Internet, the basic platform of the E-Business of Artwork. Because of various dangers in online business caused by the open Internet, the corresponding requirement of security control is put forward.

Network System. The key solution to making the Internet as the controllable and administrable built-in system is to separate the safeguarded Internet from the open, borderless and free environment, which has caused the problem of network security. Network system is the foundation of application system, so the network security becomes the high-priority problem. The main solutions are as follows:

Network Redundancy-the significant measure of dealing with the Single Point Failure of network system, will back up the critical network lines and equipment doubly or multiply. Because both the network line and equipment will reciprocally monitor and automatically adjust the operational status of each other, the network can handover and assign effectively when a length or a point fails or the network traffic changes suddenly to ensure the network to work normally.

System Isolation-it is divided into physical isolation and logical isolation. According to the network security levels, the network security boundary is delimited to make the Internet safe, for network or information media of different levels can't access to each. By adopting the VLAN technology and communication protocol, the business network and office network are divided into different application sub-networks logically.

Access control-it can effectively control the access authority and information flow of controlled sub-network or mainframe according to the principle of bilateral control and limited access on different trust domains. In terms of network object, we need to control the border and inner of network; in terms of network resources, information flow can realize the unilateral or bilateral control according to the security requirements to keep the limited access. For the access control, the most important equipment is firewall, usually placed at the entrance of the different domains, filters the input \& output network information packets and controls the information flow in accordance with corporate security policy as well as realizing the functions, such as the network address 
translation, real-time information audit and warning, etc. In addition, advanced firewall can also control the access of users based on their granularity.

Identification - it is the recognition of visitors' authority, which usually verifies their subject status by three ways: the first one is to understand the secret of a subject, such as user name the password and encryption key of user name; the second one is the items carried by a subject, such as magnetic card, IC card, dynamic password card and token card, etc.; the last one is characteristics or ability of a subject, such as fingerprints, voice, retina, signature, etc. Encryption is the most effective way to prevent eavesdropping, leakage, falsification and damage on the network to ensure the safety of information transmission.

The current encryption can be done in three levels, namely the encryption on link layer, network layer and application layer. Encryption on link layer focuses on communication link rather than information source and destination and it is transparent for top network subject. Encryption on network layer adopts the IPSEC core protocol, which has a dual function of encryption and authentication, to realize the security standards in IP layer. The virtual specialized network, established by the encryption on application layer, can make the enterprise get safely bigger return in less investment and ensure the safety of the users' application.

Safety monitoring-it looks for unauthorized network access attempts and irregularities by the way of information interception, including scanning, warning, blocking, recording, tracking, etc., so as to find the attack damage of system. As the most effective technological means against computer hacking, the network scanning and monitoring system with the characteristics, such as real-time, adaptive, active recognition and response, is widely used in all walks of life. Network scanning aims at detection and analysis on the security vulnerability of network equipment, including network communication services, routers, firewalls, E-mail, WEB servers, etc., in order to identify the network vulnerabilities that can be intruded illegally. Network scanning system forms a detailed report by detecting the vulnerability information, including location, description in detail, and suggestion improvements, so that the network can detect and manage the risk information.

Operating System. Operating system is the core of managing the computer resources, which is in charge of sending and managing messages, and scheduling storage space of equipments and all kinds of system resources. As the software platform of application system, the security of operating system(including application security and security vulnerability scanning), with universality and usability, has direct relationship with the application system.

Application Security-choosing a reliable operating system in terms of applications can avoid unsolicited softwares. Users can equip protecting and restoring software and backup them.

System Scanning-based on the safety assessment system, it divides the security risk levels of system and provides the whole security vulnerabilities checklist. Through scanning and analyzing the operating system of different versions, it will form the report of repairing the scanned vulnerabilities, protect the application program and data from theft and destruction.

Application System. Safe Storage of the Office System Document (Email): With the use of encryption, the identification system, key protection mechanism IC card and PCMCIA safety PC card, the important personal and organizational documents stored in the computer and on network servers are in a safe condition, where others cannot learn the contents of related documents even if they illegally obtain related documents or storage media disks through a multitude of means.

Safe transmission of documents and mails: the processed documents on security (including encryption, signature, identification of integrality, etc) transmitted to others can only be decoded and read by specified recipients through corresponding identification mechanism of security, such as IC card, PCMCIA PC card, and so on, which avoids the documents being intercepted and falsified in the process of transmitting or reach the stored equipment of recipients. It is mainly used in the transmission of reports and official documents.

\section{Targeted: Technique Measures on Network Security of Artwork E-commerce}

The use of network monitoring and lock monitoring. Network monitoring should be done by the network administrator, the server should record the users' access to the network resources and 
give an alarm for the illegal ones to attract the attention of network administrators with the forms of diagram, text or sound, etc. If the lawless person attempts to enter the network, the web-server will automatically record the times, If the times of illegal access reaches the setting value, then the account will be automatically locked. Through the analysis of recorded data, we can find the suspicious network activities and take measures to forestall the intrusion behaviors which may happen in the future.

The protection of the communication port and communication line. The remote terminal and communication line are weak links of security. At present, there are a variety of dedicated devices for port protection, we should choose the products with advanced technology and meet the actual needs. For the communication line, which should be buried in the ground and we should try to use the optical cables as far as possible, because there is no electromagnetic leakage caused by various of electromagnetic radiation, and its performance of anti-interference is excellent. Currently, there are two kinds of methods to restrain and prevent the electromagnetic leakage if the cables are put into use: One is the protection of conducted emission, which mainly though installing the filters with good performance on the power line and signal line to reduce the transfer impedance and the cross coupling between wires. Another one is the protection against radiation. The measures about electromagnetic shielding and the protection of interference method can be used. That is to say, By using the interference devices, a kind of pseudo noise associated with the computer system radiation is produced, which radiating to space to cover up the work frequency and information characteristics of the computer system, at the same time of the computer system on work.

Adopt the Security Control on Information Flow. Preventing the lawbreakers to determine the attack target by the method of flow and flow direction analysis.

This kind of security control includes: covering up the communication frequency, concealing the length, form and address of the message. The specific method is to fill in the message and change the transmission path. The methods of Link encryption in physical layer are usually used to hide the address of the message, as well as the encryption methods with feedback are commonly adopted to cover up the form of that.

Install a firewall with high performance. Firewall is a network barrier to prevent the hackers' access to a network organization. If the internal network will get into the Internet, a firewall must be set in the interface between the internal network and external network. The corresponding network communication monitoring system is set up at the boundary of the network to isolate the internal and external network, resist the intrusion of external network and ensure the data security of the internal network. There are two issues should be concerned in the specific applications of firewall techniques: firstly, the firewall is not an anti-virus software, while the workstation is the main way that viruses get into the network, so the anti-virus software should be installed on the workstation. Secondly, another weakness of the firewall technique is data updating between firewalls. It can't meet the request of real-time services if the delay is too long.

Adopting the Access Control. Access control will protect the information according to the processing capacity of computer system, which determines whether the access to the object from the subject is legal or not based on the predetermined rules. When the subject attempts to take use of some unauthorized resources, the access control mechanism will reject and report it to the audit trail system, which will give an alarm and record this event into log archives. The access control will set security attribute for files and database, divide its extent share and limit the usage mode of users through accessing matrix.

Adopting the data encryption technology. Data encryption technology is divided into symmetric and asymmetric key encryption technology. The former, with the same key, encrypts and decrypts data both in sender and receiver, such as the DES encryption algorithm. But in the latter, the two sides who encrypting data use different keys that have some special relation: one is used to encrypt and the other is for decrypting, like RSA encryption algorithm.

The E-Business of artwork, whose platform of electronic trading is Internet, is a new form of electronic data interchange after the SDI. The popularization of computer network and the development of the artwork market contribute to the development of E-Business of artwork. 
Therefore, the computer network security needs higher and wider requirements, including anti-virus, improving the capacity of resisting the illegal invasion of hackers, avoiding the illegal leakage during the transmission, ensuring the self-security of system, such as the stability of server, the enhancement its own resistance and eradication of any possible chances of hackers. In the important business application, firewall and data encryption technology must be used for protection; in data encryption, the most important thing is to improve the data encryption technology to get rid of any possible chances of criminals.

\section{Conclusion}

Essentially, the E-Business of artwork security is the information security on the Internet. It refers to protecting the hardware, software and data in network system of E-Business of artwork from destroying, changing and leaking accidentally of maliciously to make the system and the network service work continuously. Network security, not only a technological problem, but also a problem of management, can't be solved unless some comprehensive solutions is put forward to cope with various threats and attacks in all aspects to ensure the confidentiality, integrity, and availability of network information. So it will be very effective for the reliable and safe running to strengthen the network security management, make the relevant rules and regulations, to ensure the safety of the network.

\section{Acknowledgements}

Fund Project: Scientific research fund of education department, Yunnan province. Project Name:

《Research on the Network Security of Art E-commerce in New Media Context》. Project Number: 2015 Y347.

\section{References}

[1]Mingfen Huang. New media and the theory of western digital art .Shanghai: Xuelin Press,2009.

[2] (Netherlands) Mul,J., Translated by Yongxiong Mai. Odyssey in Cyberspace- To the virtual ontology and Anthropology. Guangxi: Guangxi Normal University press,2007.

[3]Wankang Li. An Introduction to Art Marketing. Shanghai: Fudan University Press,2005.

[4]Zhiwen Cai. Security of E-commerce.Beijing: Peking University Press,2013.

[5](UK) Roy.Ascott, Edited by Xiaoying Yuan. The Future is Now: Art, Technology and consciousness. Gold Wall Press,2012. 